\title{
Applied Innovation
}

\section{Colin Keogh}

1 University College Dublin

Potential competing interests: The author(s) declared that no potential competing interests exist.

\section{Applied Innovation refers to}

1. The process of learning innovation through the direct application of innovation tools, techniques and methods to solve real world problems in controlled, semi-controlled or uncontrolled environments such as Innovation workshops, classes, modules or programs.

2. Direct delivery, implementation and application of innovative ideas or solutions to real world problems on short (Weeks) to medium ( Months) timeframes 